\title{
The Role of the Integrated Stress Response and the Actin Cytoskeleton during Wound Healing
}

\author{
Erica Aukerman ${ }^{1}$, Miguel Barriera Diaz², Dan F Spandau ${ }^{2,3}$ \\ ${ }^{1}$ Indiana University School of Medicine; ${ }^{2}$ Indiana University School of Medicine, Department of \\ Biochemistry and Molecular Biology; ${ }^{3}$ Indiana University School of Medicine, Department of \\ Dermatology
}

\section{Background and Hypothesis:}

Chronic cutaneous wounds are a serious health concern afflicting millions of people. One of the primary factors preventing the closure of chronic wounds is the inability of keratinocytes to migrate across the wound bed. Epidermal keratinocytes migrate in a cohesive manner known as the keratinocyte collective cell migration (KCCM). Our lab has demonstrated that the integrated stress response (ISR) plays a key role in the KCCM. The ISR is initiated by stress-sensitive kinases, such as GCN2, and results in decreased global protein synthesis while preferentially increasing the translation of mRNAs encoding cytoprotective proteins. Wound repair also relies on the actin cytoskeleton, but the crosstalk between actin and the ISR is not well established. We hypothesize that the interaction between the ISR and the actin cytoskeleton is critical for $\mathrm{KCCM}$ during wound healing.

\section{Methods:}

Cutaneous wound healing was approximated in vitro using the KCCM-dependent scratch assay. Wild-type (WT) and GCN2-deleted (KO) keratinocytes were grown on coverslips, differentiated, scratched, and harvested at different times post-wounding. F-actin and vimentin (VIM) expression was monitored over time using fluorescent phalloidin-488 and immunofluorescence. In addition, WT keratinocytes were treated with actin-depolymerizing drugs and induction of ISR was measured using immunoblots.

\section{Results:}

Depolymerization of F-actin was observed along the leading edge of both wounded WT and GCN2-KO keratinocytes immediately following wounding. WT keratinocytes upregulated VIM expression at the leading edge whereas VIM expression remained unchanged in the wounded GCN2-KO keratinocytes. Treatment with latrunculin B and cytochalasin D, which both result in actin depolymerization, induced GCN2 phosphorylation in the differentiated WT keratinocytes.

\section{Conclusion and Potential Impact:}

F-actin depolymerization elicits a GCN2-mediated induction of the ISR. GCN2 and the ISR are critical components of the cutaneous wound repair process and their crosstalk with the actin cytoskeleton may serve as a novel therapeutic target in the treatment of chronic wounds. 